\title{
Applications using Open-Type Magnetic Shielding Method
}

\author{
T. Saito and T. Shinnoh* \\ Kajima Corporation, 5-11 Akasaka 6-Chome, Minato-ku, Tokyo 107-8348, Japan \\ *Kajima Technical Research Institute, 19-1 Tobitakyu 2-Chome, Chofu-shi, Tokyo 182-0036, Japan
}

There are many appliances generating electromagnetic noise around us and it is crucial to create practical electromagnetic shields in our everyday lives. The new-found method of "open-type magnetic shielding" allowing light and air to pass through its walls should provide opportunities for improving a wide range of applications. It also has features that can be used to easily, flexibly and precisely adjust shielding performance by changing the dimensions of strips and the intervals. This study reports some examples of actual applications using the open-type magnetic shielding method.

Keywords: magnetic field, magnetic shield, magnetic resonance imaging, power cable, transformer

\section{Introduction}

Shielding phenomena are interesting in the sense that they cannot be observed in a gravity field, but can be observed in a magnetic field. Around the $1600 \mathrm{~s}$, when people were unaware of the difference between gravity and magnetic forces, creating a magnetic shield using iron was described as a kind of natural magic in the early literature ${ }^{1)}$. Studies on magnetic shields using solid plates, infinitely long pipes, or sealed cubes have been extensively carried out for about a century since the 1820 s and around the latter half of the 20th century 2)-5).

There are many appliances generating electromagnetic noise around us. An environmental health monograph of magnetic-field effects was recently published by the World Health Organization ${ }^{6)}$. This study concludes it is essential to shield ourselves in practice from electromagnetic fields in our everyday lives.

A new-found method of "open-type magnetic shielding" allowing light and air to pass through its walls should provide opportunities for improving a wide range of applications ${ }^{7)}$. It has been determined that a wall composed of aligned strips with gaps can achieve almost the same magnetic-shielding effect as a conventional solid plate ${ }^{8}$. It also has a feature where the shielding performance can easily, flexibly and precisely be adjusted by changing the dimensions of the strips, the number of layers for each strip, and the intervals between strips. For example, it has been reported that double layers for each strip with a double gap can achieve almost the same shielding performance as a single layer with a single gap. It has also been reported that the numerically analyzed results for shielding about a wall with open-type magnetic shielding coincide well with the experimental results when real data on a non-linear B-H curve was used ${ }^{9}$.

This study reports some examples of actual applications using an open-type magnetic shielding method. A detailed example for an MRI room is reported in the case of a DC magnetic field, and another is reported for a power cable in the case of an $\mathrm{AC}$ magnetic field.

\section{Open-type magnetic shielding method}

\subsection{Magnetic shielding wall}

Fig. 1(a) illustrates a magnetic field/flux generated by a current in an electric wire and a plate-like magnetic material used for a magnetic shield. For shielding magnetic fields to reduce leakage to the space to be shielded, we have generally placed a plate-like high permeability material between the source generating the magnetic field and the space to be shielded, because magnetic flux enters into it and creates a detour ${ }^{10)}$.

In contrast to this conventional method, Fig. 1(b) illustrates the open-type magnetic shielding method. Strip-like materials aligned in a line with gaps are used. There can be air or a vacuum in the gaps. Even though this structure can pass air, light, or objects that are smaller than the gaps, it can still provide a shield for a magnetic field. Thus far, it has been reported that a single wall composed of aligned strips in a line using this open-type magnetic shielding method can achieve almost the same magnetic shielding effect as the conventional solid-plate method ${ }^{8)}$.
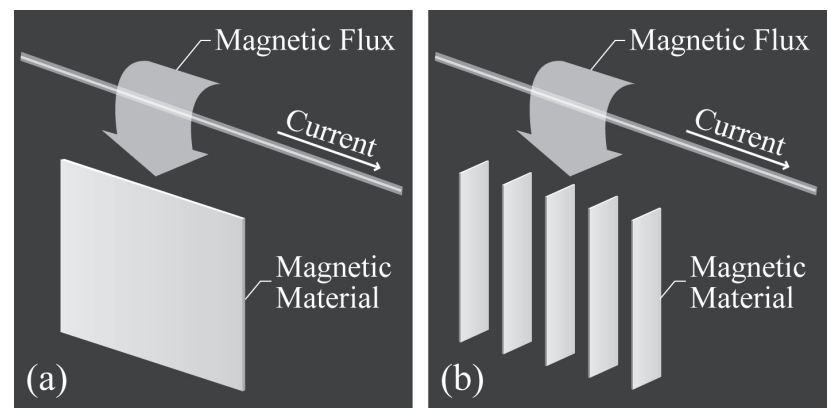

Fig. 1 (a) Conventional magnetic shielding method; solid plate of magnetic material without any gaps has been used. (b) Open-type magnetic shielding method; aligned strips of magnetic material with gaps. Light and air can pass through this magnetic shielding wall. 


\subsection{Magnetic shielding box}

Fig. 2(a) illustrates a magnetic shielding box using the conventional method. To efficiently shield a uniform magnetic field from the outside, the space to be shielded has generally been enclosed by high permeability magnetic materials without any gaps/holes, so the magnetic flux enter into them, make detours, and do not penetrate inside. Magnetic plates have size limitations necessitating joints for larger structures, and they require special connecting materials such as flat bars and $\mathrm{L}$ angles to magnetically connect the plates ${ }^{11), 12)}$.

In contrast to the conventional method, frame-like materials aligned with gaps have been proposed as illustrated in Fig. 2(b) 13). The total structure consists of six faces using strips of high permeability materials which are orthogonal to each other. Air or a vacuum can fill the gaps. Therefore, this structure can pass air, light, or objects that are smaller than the gaps, while continuing to shield magnetic fields. It has been reported that shielding performance of an open-type magnetic shielding box is better than that of the conventional sealed box when they weigh the same.
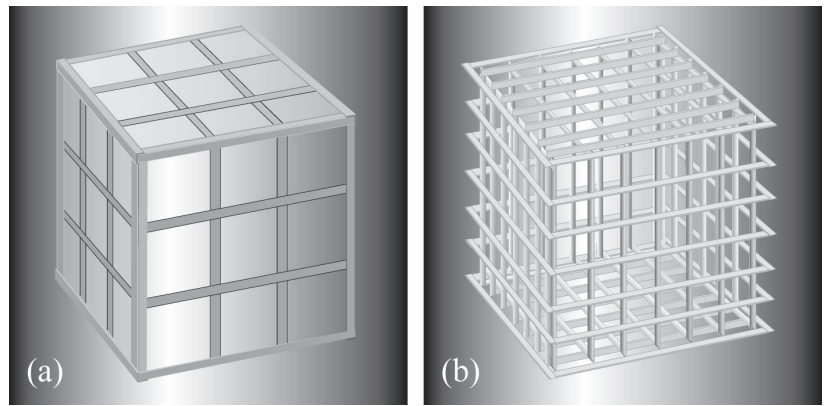

Fig. 2 (a) Magnetic shielding box with conventional method; the space to be shielded has been enclosed by magnetic material without any gaps. (b) Magnetic shielding box with open-type method; each surface has orthogonal strips of high permeability material with gaps.

\subsection{Features of open-type magnetic shielding method}

The six main features of the open-type magnetic shielding method that have been reported are:

1) It can pass air, light, heat, or objects that are smaller than the gaps, while continuing to shield magnetic fields.

2) The shielding performance for a wall is almost the same as that for a conventional solid wall when the weight is the same.

3) The shielding performance of a box is better than that of a conventional sealed box when the weight is the same.

4) The shielding performance is easy to precisely adjust by changing the kinds of magnetic materials, the dimensions of the strips, the number of layers for each strip, and the intervals between strips.

5) The values calculated by computer analysis are easy to correlate to an extent with the measured values obtained from manufacturing or construction.

6) The shielding wall does not become thicker, because almost the same shielding performance can be achieved with 0 and 90 degrees of rotation around the axis parallel to the length direction.

\section{Example of Application for DC Magnetic Field}

\subsection{A field of application}

MRI is a popular medical device utilizing a very strong DC magnetic field such as 3 Tesla. To date, in many cases walls have been shielded magnetically by plate-like material; thus, the room has had a closed atmosphere. Therefore, patients suffering from claustrophobia have been hesitant or not able to undergo necessary examinations.

However, if the open-type magnetic shielding method is applied to MRI rooms, walls can be made transparent. Thus, it can considerably alleviate the psychological feeling of being boxed in. Fig. 3 is a photograph of an application for an MRI room. Strips horizontal to the ground in the wall provide an open-type magnetic shield. The standard gap between these is $300 \mathrm{~mm}$. This type of room is called a transparent MRI room or a see-through MRI room. About 30 have so far been installed throughout the world.

The medical verification results about the effects of these transparent MRI rooms reported improvement in patients feelings and well being ${ }^{14)}$,15). Recently, another method of magnetic and RF shielding was reported in which piled up square cylinders made of electrical steel sheet and aluminum were used ${ }^{16)}$.

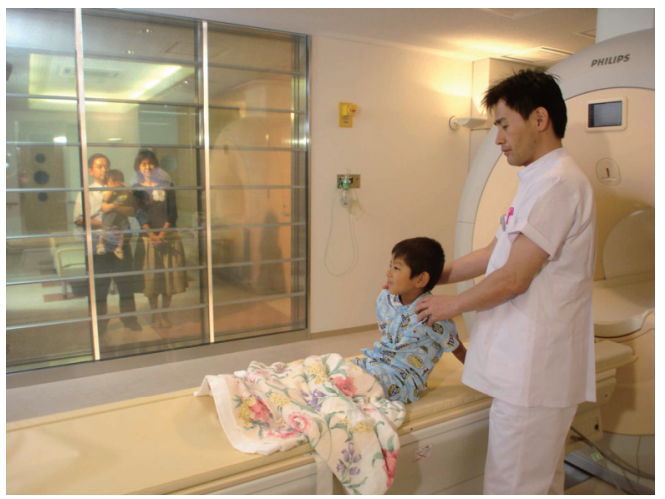

Fig. 3 Photograph of an application for MRI rooms. This type of room is called a transparent MRI room or see-through MRI room.

\subsection{Development process for actual application}

Three steps of development including different sized experimental models for an MRI room were carried out as follows:

1) Approximately 500 experiments were carried out on small box models in order to understand 
fundamental characteristics and to evaluate the accuracy of numerical analysis 17). The dimensions of the shielding box were $900 \mathrm{~mm}(\mathrm{~W})$ $\times 900 \mathrm{~mm}(\mathrm{~L}) \times 900 \mathrm{~mm}(\mathrm{H})$. There was an electromagnet inside the box, and the magnetic flux density outside the box was measured. The average error between the experiment and numerical analysis was approximately $5 \%$.

2) Approximately 100 experiments were conducted on medium-sized-room models to take into consideration joint parts, the door, and detailed points. The dimensions of the shielding model were $2810 \mathrm{~mm}(\mathrm{~W}) \times 3500 \mathrm{~mm}(\mathrm{~L}) \times 1700 \mathrm{~mm}(\mathrm{H})$, which was intended to represent almost half of a real room. Another electromagnet was manufactured and located inside the models. Numerous conditions for the length of strips, the number of layers, and the number of joint parts were evaluated, and the magnetic flux density outside the room was measured.

3) Approximately 80 experiments were undertaken on fully sized room models for the final evaluation. An actual MRI magnet was used and located inside the room. The dimensions of the shielding model were $4515 \mathrm{~mm}(\mathrm{~W}) \times 5887 \mathrm{~mm}$ $(\mathrm{L}) \times 3200 \mathrm{~mm}(\mathrm{H})$. Numerous conditions on the length of the strips and the number of layers were evaluated and the magnetic flux density outside the room was measured.

In addition, grain-oriented electrical steel sheet was used in all experiments as strips for open-type magnetic shielding method. Fig. 4 shows the material data. It was reported that the direction of high-permeability should be parallel to the length direction of strips and magnetic field ${ }^{8)}$.

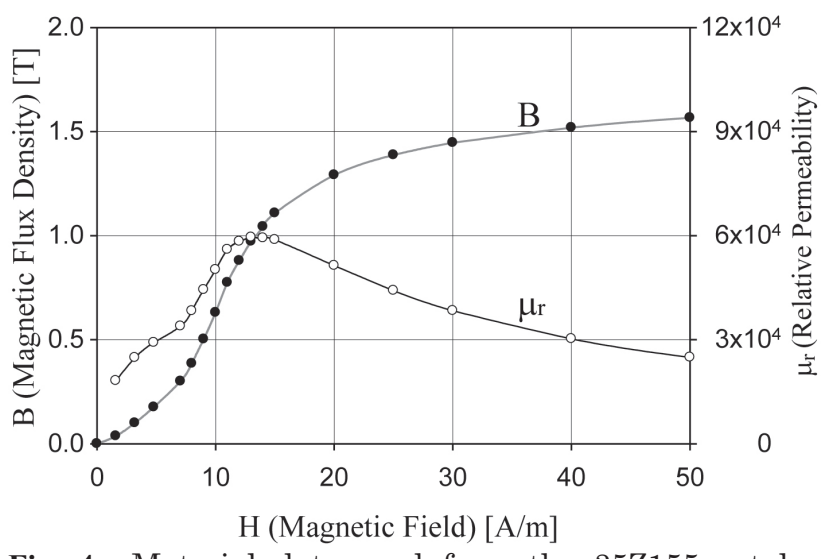

Fig. 4 Material data read from the $35 \mathrm{Z} 155$ catalog which is a grain-oriented electrical steel sheet manufactured by Nippon Steel Corporation.

\subsection{Example of application in MRI room}

An application example of the open-type magnetic shielding method at an MRI room for Ueda neurosurgery in Miyazaki, Japan is introduced.
Fig. 5 is a photograph of the inside of an MRI room. The MRI equipment is a Signa HDe Second Edition 1.5 $\mathrm{T}$ made by GE. It was necessary to reduce the leakage to under $0.5 \mathrm{mT}$ outside the wall to the right in order to obey the JIS Z4951 translating IEC60601-2-33 which the guideline of Food and Drug Administration in United States of America is also compliant with ${ }^{18)}$. Fig. 6 has a top view of the MRI room. An open-type magnetic shield was applied to the blue part. The bright and open atmosphere was achieved allowing by the outside light into this room through this magnetically shielded wall. The other wall, floor, and ceiling are not magnetically shielded. The red line indicates the contour of $0.5 \mathrm{mT}$ calculated by $3 \mathrm{D}$ and nonlinear numerical analysis with a computer, which includes the earth magnetism around this room.

The magnetic shielding strips were horizontal to the ground. Grain-oriented electrical steel sheets that were $50 \mathrm{~mm}(\mathrm{~W}) \times 3260 \mathrm{~mm}(\mathrm{~L}) \times 7 \mathrm{~mm}(\mathrm{t})(0.35 \mathrm{~mm} \times$ 20 layers) were aligned with $300 \mathrm{~mm}$ gaps. These strips were sandwiched between two RF shielding glasses that were highly transparent. This magnetic and $\mathrm{RF}$ shielded wall was $3260 \mathrm{~mm}(\mathrm{~L}) \times 2600 \mathrm{~mm}(\mathrm{H}) \times 144$ $\mathrm{mm}(\mathrm{W})$. The degree of RF shield was over $100 \mathrm{~dB}$ from $10 \mathrm{MHz}$ to $100 \mathrm{MHz}$.

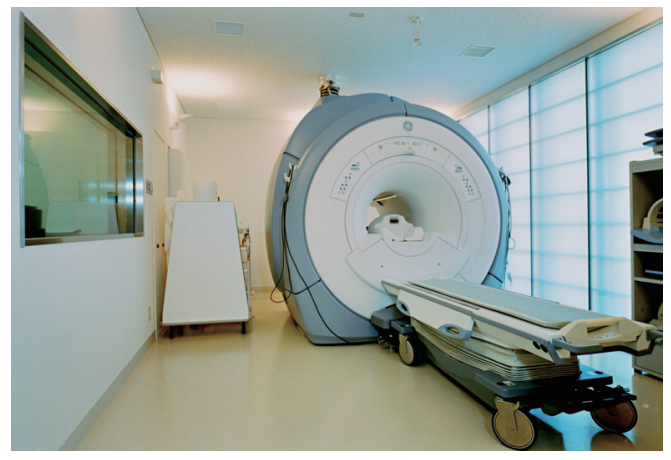

Fig. 5 Photograph of an application for the MRI room at Ueda neurosurgery. The open-type magnetic shield is being applied on the right.

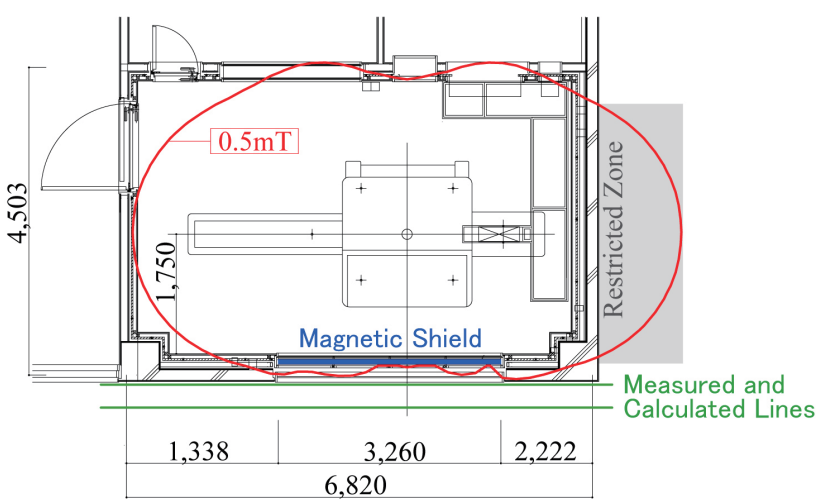

Fig. 6 Top view of the MRI room. Only the blue part was magnetically shielded using an open-type magnetic shielding method. The red line indicates the contour of $0.5 \mathrm{mT}$ calculated by $3 \mathrm{D}$ and nonlinear numerical analysis. The two green lines are measured and calculated lines in the Fig. 7. 
Fig. 7 compares the measured and calculated values in the two lines outside the MRI room and parallel to the magnetically shielded wall, which are the two green lines in the Fig. 6. The horizontal separation distance between these lines and the wall surface were $0 \mathrm{~mm}$ and $300 \mathrm{~mm}$. The heights of the lines coincided with the center of the MRI magnet. The two main findings from these results are as follows:

1) Values for magnetic flux density at maximum was reduced from $0.97 \mathrm{mT}$ without magnetic shielding to $0.39 \mathrm{mT}$ with the shield measured at $0 \mathrm{~mm}$ from the wall surface. This was reduced from $0.45 \mathrm{mT}$ without a shield to $0.21 \mathrm{mT}$ with a shield $300 \mathrm{~mm}$ from the wall surface.

2) The calculated values were found to be in good agreement with those measured. The maximum values $0 \mathrm{~mm}$ from the wall surface were $0.39 \mathrm{mT}$ for measurement and $0.37 \mathrm{mT}$ for calculation. The maximum values $300 \mathrm{~mm}$ from the wall surface were $0.21 \mathrm{mT}$ for measurement and 0.22 $\mathrm{m} \mathrm{T}$ for calculation.

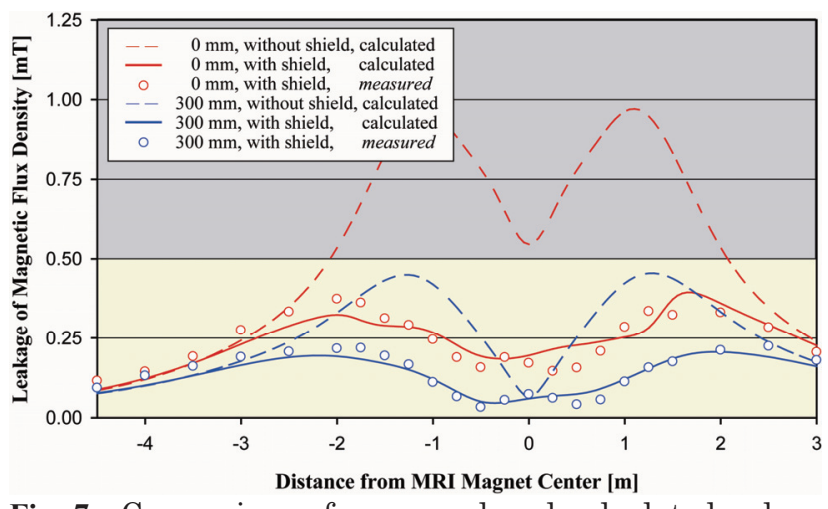

Fig. 7 Comparison of measured and calculated values at distance represented in Fig. 6 by the two green lines outside the MRI room parallel to the magnetically shielded wall.

\section{Example of Application for AC Magnetic Field}

\subsection{Fields of application}

Fig. 8 is a photograph of an application for power cables. Fig. 9 is a photograph of an application for a transformer. They generate AC magnetic-field noise.

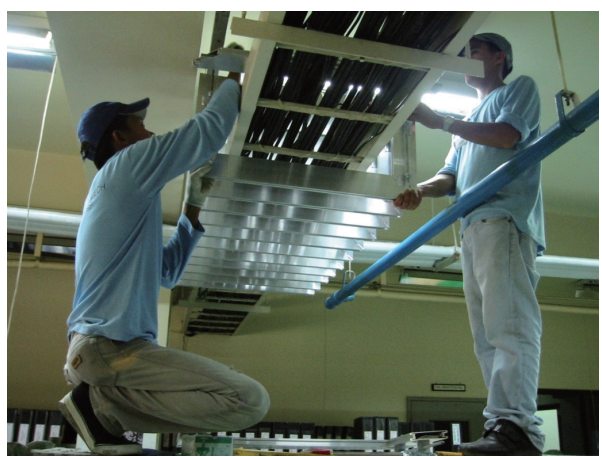

Fig. 8 Photograph of an application for power cables.

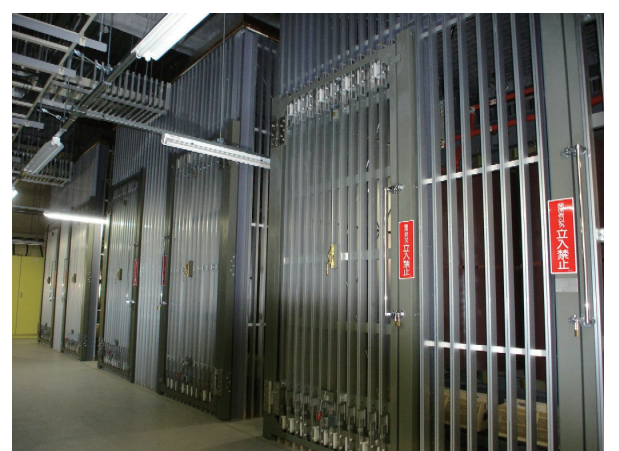

Fig. 9 Photograph of an application for transformers.

The conventional shielding method uses tube or box type magnetic material enclosures around them which do not dissipate heat and make maintenance access difficult. Thus, in many cases, large areas of room ceilings or floors carrying AC electricity are sealed with plate-like magnetic material.

However, if open-type magnetic shielding is applied to these applications, the structure is shielded from magnetic fields and also has sufficiently wide gaps to dissipate heat and enable maintenance.

\subsection{Development process before actual application}

This subsection introduces the development process for power cables. Fig. 10 shows an experimental system using an open-type magnetic shield, which is composed of power cables, a magnetic shield, a measuring device with a sensing probe, a fine moving system for the probe, and a computer for control. The length of power cable is $7850 \mathrm{~mm}$, and that of the magnetic shield is $4500 \mathrm{~mm}$. The open-type magnetic shield was composed of aligned frame units that had a magnetically closed loop structure. Each magnetic shield unit is made of four strips like an Epstein frame. In total, about 100 cases were carried out in this experiment. The experimental conditions for the positions of the power cables, the electric current, the number of layers for magnetic materials, and the gap between the magnetic shielding units can be seen in the figure.

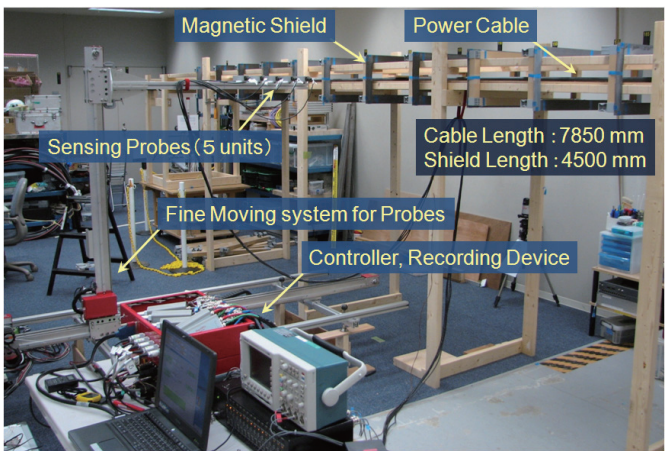

Fig. 10 Photograph of experimental system for power cables. In this case, the thickness of the magnetic shielding units is $1.75 \mathrm{~mm}$, the pitch of these units is $450 \mathrm{~mm}$ and the width of strip made of grain-oriented electrical steel sheet is $50 \mathrm{~mm}$. 
Fig. 11 shows a cross section of the experimental model. The dimensions of each magnetic shielding unit were $900 \mathrm{~mm}(\mathrm{~W}) \times 300 \mathrm{~mm}(\mathrm{H}) \times 1.75 \mathrm{~mm}(\mathrm{t})(0.35 \mathrm{~mm}$ $\times 5$ layers) and each strip was $50 \mathrm{~mm}$ wide. The units were aligned in $150 \mathrm{~mm}$ pitches. In this case, the gaps between each unit was $148.25 \mathrm{~mm}$ subtracting $1.75 \mathrm{~mm}$ of thickness from $150 \mathrm{~mm}$ of pitch. All experimental models were also calculated by using 3D and nonlinear numerical analysis with a computer. The area in light blue was measured and calculated. The $\mathrm{z}$ cordination of this area was $75 \mathrm{~mm}$ which represented that it is located in the middle of the two central shielding units.

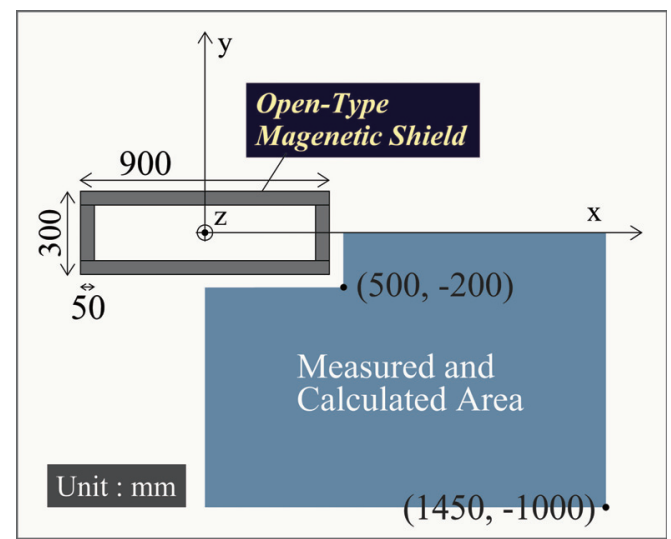

Fig. 11 Cross section of an experimental model. The area in light blue was measured and calculated.

Fig. 12 indicates a result of numerical analysis in a case. An AC magnetic field was generated from three power cables in the center of this model. The value of the electric current was $1273 \mathrm{Ap}^{-p}$ (=450 Arms) each. The frequency was $50 \mathrm{~Hz}$. The distance between each cable was $100 \mathrm{~mm}$. The phase of each cable was 0,120 , and 240 degrees, which was three-phase alternating current. The contour lines of $25 \mu \mathrm{Tp}$-p with/without a magnetic shield are shown in Fig. 12. It is found that the magnetic field around the power cables was reduced.

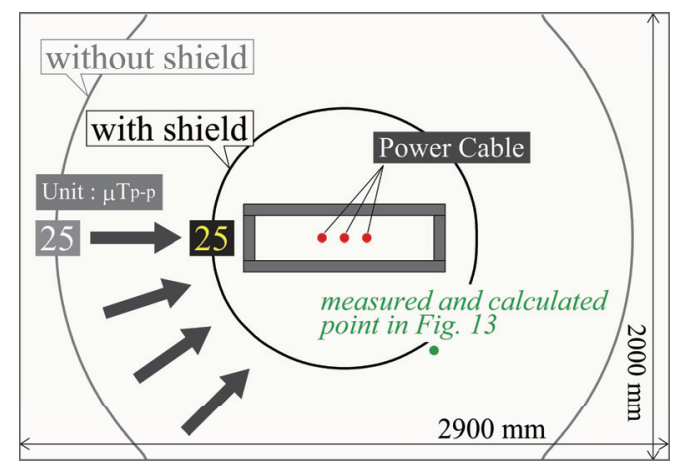

Fig. 12 A result of numerical analysis. The distance between each cable was $100 \mathrm{~mm}$. The current was 1273 Ap-p, $50 \mathrm{~Hz}$ each and the phase of each current was 0 , 120 , and 240 degrees. The dimensions of each magnetic shielding unit were $900 \mathrm{~mm}(\mathrm{~W}) \times 300 \mathrm{~mm}(\mathrm{H}) \times 1.75$ $\mathrm{mm}(\mathrm{t})(0.35 \mathrm{~mm} \times 5$ layers $)$ and each strip was $50 \mathrm{~mm}$ wide. The units were aligned in $150 \mathrm{~mm}$ pitches.
As a result of calculation, the magnetic flux density in the light blue area of Fig. 11 was $878.8 \mu \mathrm{Tp}-\mathrm{p}$ at maximum and $13.7 \mu \mathrm{Tp}-\mathrm{p}$ at minimum in the case without shield and $346.4 \mu \mathrm{Tp}-\mathrm{p}$ at maximum and 3.4 $\mu \mathrm{Tp}-\mathrm{p}$ in the case with shield. The shielding factor that is magnetic flux density without shield divided by magnetic flux density with shield at each point was 5.7 at maximum and 2.5 at minimum. This represents that magnetic flux density generated by power cables outside the open-type magnetic shielding units is reduced to approximately $1 / 3$ to $1 / 6$ in this case.

Fig. 13 compares the measured and calculated values at the green point in Fig. 12. Its coordination is $\mathrm{x}$ $=400 \mathrm{~mm}, \mathrm{y}=-500 \mathrm{~mm}$ and $\mathrm{z}=75 \mathrm{~mm}$. They were found to be in good agreement both for the amount and phase. In this case, the average error between the experimental and numerical analyses was approximately $5 \%$ without the shield and $8 \%$ with it.

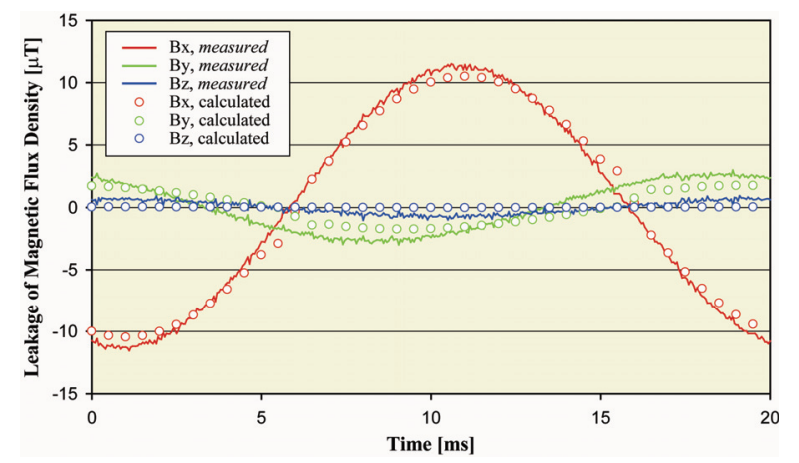

Fig. 13 Comparison of measured and calculated values for the magnetically shielded state. The directions of $x$, $\mathrm{y}$ and $\mathrm{z}$ are described in Fig. 11.

\subsection{Application of power cable}

This subsection introduces the application of a power cable for a factory in Southeast Asia. There is an office directly above a room where electricity with approximately $70 \mathrm{CV}$ power cables is used in the factory. The displays of the CRT monitors were vibrating due to magnetic noise. The AC magnetic flux density was measured in the office. Fig. 14 plots the distribution and contour of magnetic flux density for magnetic noise 700 $\mathrm{mm}$ from the office floor. Red represents high density of magnetic flux and blue represents low density of it. It was found that maximum magnetic noise as strong as $14.9 \mu \mathrm{Tp}$-p was generated along the power cables.

The frame units using the open-type magnetic shield were installed as shown in Fig. 8. The magnetic material was a grain-oriented electrical steel sheet. The dimensions of each unit were $710 \mathrm{~mm}(\mathrm{~W}) \times 310 \mathrm{~mm}(\mathrm{H})$ $\times 7 \mathrm{~mm}(\mathrm{t})(0.35 \mathrm{~mm} \times 20$ layers $)$ and each strip was 50 $\mathrm{mm}$ wide. The units were aligned in $150 \mathrm{~mm}$ pitches. The gaps between each unit including case made of surface-treated alminium for insulation was $128 \mathrm{~mm}$, becase the thickness of the cases is $22 \mathrm{~mm}$. In addition, CV cables with the same current and different phase were bound together in a set. 
Fig. 15 plots the distribution and contour for the magnetic flux density of magnetic noise $700 \mathrm{~mm}$ from the office floor after these countermeasures. The color scale is the same as Fig. 14. It was found that the area in red and yellow had disappeared and the maximum magnetic noise was only as strong as $2.8 \mu \mathrm{Tp}-\mathrm{p}$. Finally, the problem with the displays' vibrating was completely solved. Fig. 16 has the contour distribution of magnetic-reducing effect $R$, which means the magnetic flux density was reduced to $1 / \mathrm{R}$. $\mathrm{R}$ at maximum was approximately 36 in this case.

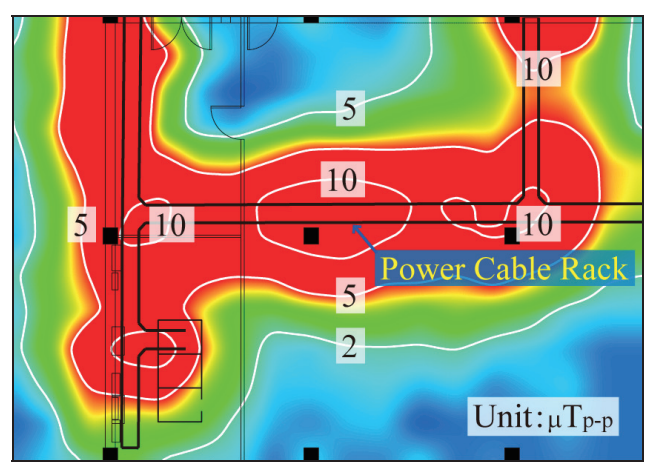

Fig. 14 Contour distribution of magnetic flux density for magnetic noise at $700 \mathrm{~mm}$ on the office floor before countermeasures.

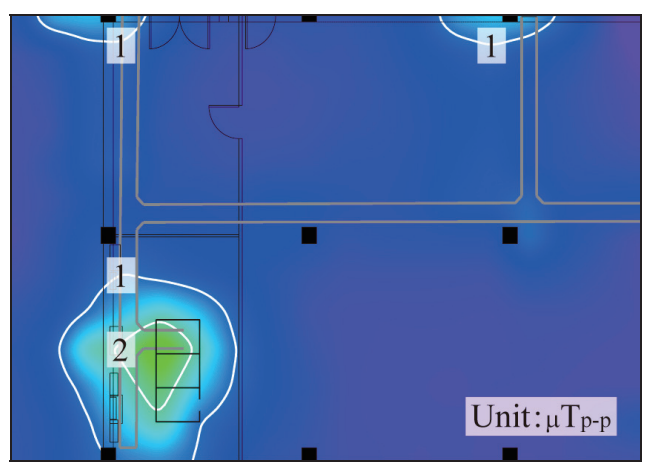

Fig. 15 Contour distribution of magnetic flux density for magnetic noise at $700 \mathrm{~mm}$ on the office floor after countermeasures.

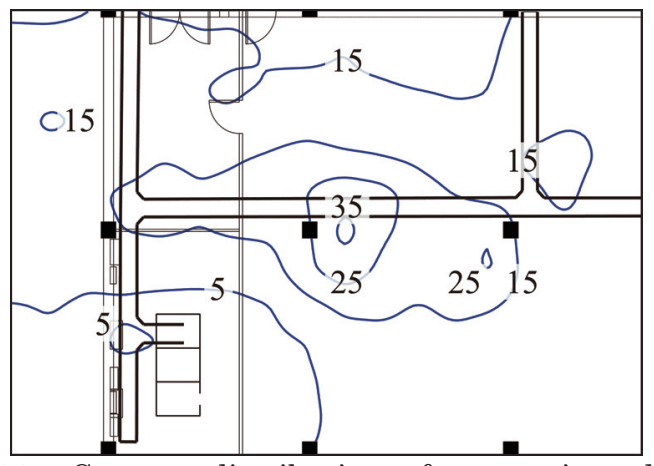

Fig. 16 Contour distribution of magnetic reducing effect $R$, which means the magnetic flux density is reduced to $1 / \mathrm{R}$.

\section{Conclusion and Discussion}

Examples of actual applications using open-type magnetic shielding method were reported. A detailed example for an MRI room was reported for a DC magnetic field and another was reported for power cables for an AC magnetic field. The shielding performance simulated by numerical analysis for the open-type magnetic shielding method is precise enough for practical use. This means highly reliable magnetic shielding specifications can be designed for many purposes under any criteria.

Magnetic shields are expected to be utilized in the future all over the world because the open-type magnetic shielding method allows light and air to pass through, which provides opportunities for a wide range of applications. Most interests are applications making use of the progression to stronger/weaker magnetic fields with greater and wider frequencies. Open-type magnetic shielding is thought to be useful in practice in many cases. Further research and development in a variety of fields is expected.

Acknowledgements: The author would like to thank Kajima Corporation and Nippon Steel Corporation for their support.

\section{References}

1) J. B. Della Porta: De miraculis magnetis, in Magie natulis, Libri VII (1589).

2) A. W. Rücker: Phil. Mag., 37, 95 (1894).

3) W. Esmarch: Annalen der Physik, 39, 1540 (1912).

4) D. A. Cohen: J. Appl. Phys., 38, 1295 (1967).

5) A. J. Mager: IEEE Trans. Magn., 6, 67 (1970).

6) World Health Organization: Extremely low frequency fields, Environmental Health Criteria 238 (2007).

7) T. Saito, Tokkyo Koho JP 3633475 (2005).

8) T. Saito: IEEE Trans. Magn., 44 (no. 11), 4191 (2008).

9) T. Saito: Proc. International Symposium on Nonlinear Theory and its Applications, 572 (2008).

10) T. J. Sumner, J. M. Pendlebury, and K. F. Smith: J. Phys. D: Appl. Phys., 20, 1095 (1987).

11) B. J. Patton and J. L. Fitch: J. Geophys. Res., 67 (no. 3), 1117 (1962).

12) V. O. Kelhä: Biomagnetism. Walter de Gruyter \& Co., Berlin • New York, 33 (1981).

13) T. Saito: IEEE Trans. Magn., 45 (no. 10), 4640 (2009).

14) N. Aoyama: Jpn. J. Magn. Res. Med. (in Japanese) 27, 438 (Suppl), (2007)

15) Y. Tutui: 24th Annual Conf., Session Code: 67 (The Japan Association of Radiological Technologists, Sapporo, 2008).

16) S. Hirosato, K. Yamazaki, Y. Haraguchi, K. Muramatsu, A. Haga, K. Kamata, K. Kobayashi, A. Matsuura and H. Sasaki: IEEE Trans. Magn., 45 (no. 10), 4636 (2009).

17) H. Hirano, T. Saito, T. Shinnoh, M. Fujikura and K. Chikuma: IEEE Trans. Magn., 45 (no. 10), 4648 (2009).

18) H. Kawamitsu, T. Dobashi, T. Miyachi, H. Sugimoto, T. Doi, H. Muranaka, A. Ogura, T. Matsuda and T. Okuaki: Jpn. J. Rad. Tech. (in Japanese), 64 (no. 12), 1575 (2008).

Received Oct. 21, 2009; Revised Dec. 24, 2009; Accepted Feb. 10,2010 